\title{
Familia y Pobreza dura
}

\author{
Germán Rozas ${ }^{1}$
}

\section{Resumen}

Un tema de alta complejidad hoy en día es la superación de la pobreza, no obstante existe un segmento de la misma que aparece especialmente resistente, la Pobreza Dura. Esta área es refractaria a las políticas sociales que ponen el acento en el aspecto económico. Surgen así otras dimensiones a considerar, entre las cuales la familia es una fundamental. Sin embargo particularmente las variables psicosociales de la familia. Se plantea, entonces la hipótesis, que dichos aspectos originados en una época histórica anterior conforman matrices fundacionales de la familia pobre en Chile. Estas matrices se despliegan en la actualidad en un enfrentamiento con la modernidad. Dicha contradictoria dinámica profundiza la situación provocando un tipo de familia asociada a la pobreza dura.

Palabras Claves: Pobreza Dura, Familia Pobre, Matrices Psicosociales.

\section{Abstract}

A highly complex subject is to surpass poverty; However, there exists a segment that it is specially tough: Extreme Poverty.

This is an obstinate social political area with an accent in the economic aspect. Following this arise other dimensions are to be considered, among them, the family, which is fundamental and, particularly, the psychosocial variables of the family.

This gives ground to the hypothesis that those aspects derive from a historic time which conform the mother of the foundation of the Chilean poor family. This mold unfolds today a confrontation between past and modern times. This dynamic contradiction deepens the situation inciting a type of family associated to extreme poverty.

\section{I.-Antecedentes pobrezadura}

El término de "Pobreza Dura" constituye un concepto en formación. Se aproxima a identificar un segmento de la población pobre que tradicionalmente y en diferentes contextos se le ha denominado indigentes, pobreza crítica o extrema pobreza.

Estos últimos conceptos se encuentran asociados a la forma tradicional de medición de la Pobreza basada en el Método del Ingreso, consistente en la comparación de la canasta familiar con el ingreso, donde los pobres críticos son aquellos cuyos ingresos son menores que el resto de los pobres. Este enfoque tan difundido y tan utilizado por los gobiernos para la elaboración de politicas sociales tiene fundamentalmente como soporte la dimensión económica.

No se abandona dicha dimensión económica, al referirse a Pobreza Dura, pero existe un esfuerzo en los especialistas delárea por incorporar otras dimensiones que permitan entender más integralmente el fenómeno. Surgen así aspectos significativos como la calidad de las relaciones al interior de la familia, las limitaciones y potencialidades del desarrollo de las personas en pobreza, 
el entorno social e institucional que activa o deprime la reproducción de la pobreza misma. En este artículo justamente se intenta mostrar factores relevantes de la Pobreza Dura en torno al ámbito de la Familia.

Por otro lado la emergencia y necesidad de un concepto más amplio de Pobreza Dura surge en cierto sentido, del mismo análisis de los resultados cuantitativos del Método del Ingreso, que indican que pese a la disminución de la pobreza (pobreza no indigente), la mitigación de la indigencia no ha ocurrido de la misma forma, no ha seguido con la misma celeridad la curva descendente general. Se aprecia una población que vive en pobreza critica que tiende a mantenerse como tal, a no sufrir grandes modificaciones. (ver cuadro $\left.\mathrm{n}^{\circ} 1\right)$.

Al respecto el cuadro muestra que en el periodo 90-96 la pobreza no indigente disminuyó un 8,5\% y
El análisis nos lleva a revisar ciertos factores involucrados. Desde la perspectiva de la Psicología Social en relación a la dimensión individual aparecen ciertas dificultades personales asociadas a la autoestima, la desesperanza aprendida que tienen una incidencia directa en la habilitación de las personas en el campo laboral o en el desarrollo de iniciativas. Estos aspectos influyen por otra parte en el seno de la familiar afectando las relaciones padres e hijos, y produciendo una espiral negativa de reproducción de incapacidades.

De la misma forma variables como el entorno poblacional, las dificultades de acceso a la salud, los déficit de establecimiento educacionales, etc. son también aspectos relevantes como los anteriormente mencionados, (Rozas, 1998).

\section{Cuadro №1}

EVOLUCIÓN DE LA POBREZA EN CHILE

\begin{tabular}{llrrc}
\hline AÑO & $\mathbf{1 9 9 0}$ & $\mathbf{1 9 9 2}$ & $\mathbf{1 9 9 4}$ & $\mathbf{1 9 9 6}$ \\
\hline Pobreza & $27 \%$ & $23,1 \%$ & $20 \%$ & $18,5 \%$ \\
Indigencia & $13 \%$ & $8,9 \%$ & $8,5 \%$ & $6,5 \%$ \\
Pobreza Total & $40 \%$ & $32,7 \%$ & $28,5 \%$ & $25 \%$ \\
\hline
\end{tabular}

Fuente: Cepal/Mideplan

Casen 1990-92-94-96

la pobreza indigente disminuyó $6,5 \%$. Como se señalaba más arriba la disminución no es en la misma proporción. Más profunda, es dicha diferencia al comparar los años 1992-1994, donde la pobreza no indigente disminuye un $3.1 \%$, y la pobreza indigente solo alcanza una disminución de $0.4 \%$.

Es alli donde en el campo de las ciencias sociales, surge la interrogante sobre las causas que expliquen esta situación, ¿por qué disminuye la pobreza y no disminuye con la misma rapidez la indigencia?, al parecer están presentes aquí otros fenómenos.

\section{II.-Relación pobrezay familia}

La CEPAL (1994) en torno al tema de la pobreza pone el acento en el campo de la familia, categorizando los núcleos duros de la pobreza. Definidos como aquellas familias u hogares que presentan Jefatura femenina, sin cónyuge, con tres o más hijos, cuyos ingresos se encuentran bajo de la linea de Pobreza.

No se trata de desmerecer la jefatura femenina, más bien el problema reside en que la discriminación económica dirigida hacia la mujer provoca un 
promedio menor del ingreso familiar que si el jefe de hogar es el hombre, por lo mismo esta realidad hace referencia a un extremo de vulnerabilidad social.

Los antecedentes en nivel latinoamericano, sobre mediciones de la pobreza muestran que el porcentaje de mujeres jefas de hogar es mucho mayor en la población que se encuentra bajo la linea de indigencia.
A modo de ejemplo, en Venezuela (Cepal, 1992), el $40 \%$ de familias indigentes tienen jefatura femenina, en cambio en los pobres no indigentes dicha calidad se encuentra en algo más de un 20\% . Es decir, prácticamente el doble de familias con jefatura femenina se encuentran en el segmento indigente en relación al segmento no indigente. Estos antecedentes se repiten de manera similar en los diferentes países de América Latina (Ver Cuadro $\mathrm{N} 2$ ). Lo mismo ocurre y tal vez con más profundidad en el caso del sector rural.

\section{Cuadro $\mathrm{N}^{\circ} 2$}

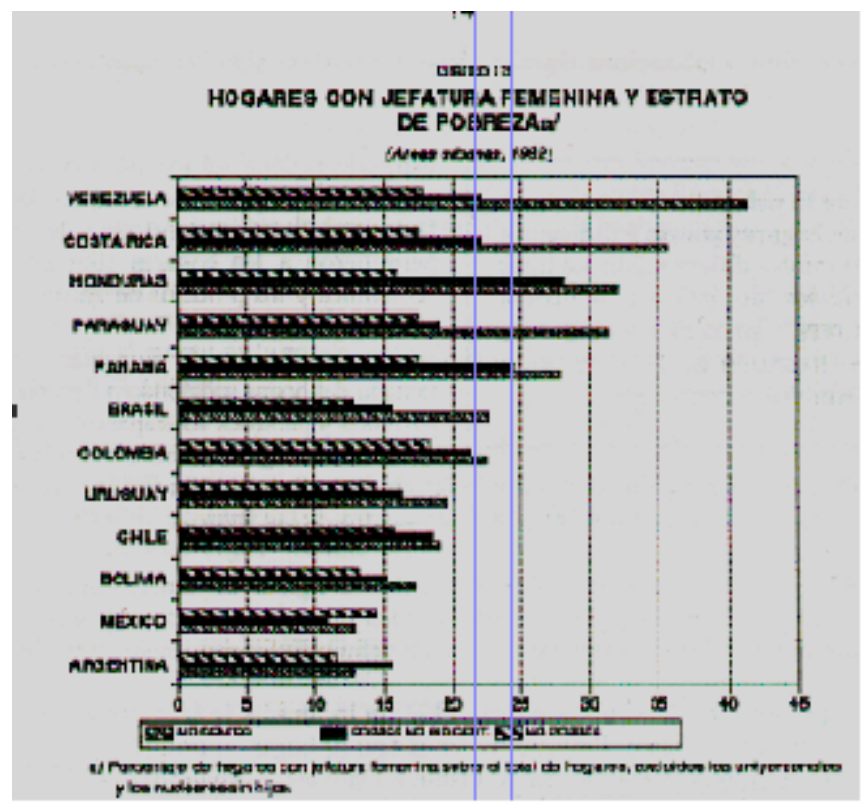


Complementando los antecedentes, sin perder la referencia de la familia, el porcentaje de jóvenes desocupados de 15 a 29 años en 1996, comparando el primer quintil (jóvenes más pobres) y el quinto quintil (jóvenes más acomodados) se observa que el primero alcanza la tasa mas alta de desocupación de un $23,2 \%$ y el quinto grupo sólo llega al 3,5\% (Mideplan, 1997).

Luego relacionando estos resultados con el tema de la educación, en el sentido de que el proceso formativo, especialmente la capacitación postsecundaria, son dinámicas que permiten la integración a la sociedad, según el INJ, los jóvenes que ingresan a realizar estudios postsecundarios del primer quintil son el 11\% en franca diferencia con los jóvenes del último quintil quienes ingresan en un 30,2 \%. (Primer Informe Nacional de Juventud, INJ, 1994).

¿Que hacen estos jóvenes entonces?, Algunas pistas ofrece el diagnóstico del Plan de Desarrollo Comunal de Peñalolén. (cuadro N³). En

\section{III.-Familiay pobrezadura: circulo vicioso}

La familia en Pobreza Dura se encuentra atrapada en una especie de laberinto social donde a cada movimiento pareciera hundirse más y más. Los distintos actores que van conformando la familia, padre, madre, hijos, abuelos, amigos presentan inhabilidades sociales que en distintos momentos les impiden salvar obstáculos fundamentales para su integración social. Con bajo niveles educacionales, limitaciones psicosociales, diminuida perseverancia, alcanzan sólo labores esporádicas y mal remuneradas; sin ingresos suficientes se torpedean condiciones esenciales de calidad de vida, vivienda, alimentación, recreación, vestuario; lo que a su vez termina horadando la salidad del sistema, impactando a los niños. Estos últimos sin herramientas intelectuales, culturales, sociales ven fragmentado su proyecto vital, reconstituyendo el ciclo de la pobreza.

\begin{tabular}{|lcc}
\hline \multicolumn{3}{c|}{$\begin{array}{c}\text { Cuadro №3 (cuadro Peñalolén) } \\
\text { Actividad delos) ovenes ComunadePeñalolen 1993 }\end{array}$} \\
\hline ACTIVIDAD & Número & $\%$ \\
\hline Estudia & 13.810 & 37.10 \\
Cesante & 7.973 & 21.43 \\
Trabaja & 6.884 & 18.24 \\
Guehacer Hogar & 6.032 & 16.22 \\
Sin actividad o busca trabajo & 2.439 & 6.53 \\
TOTAL & 37.233 & 100.00 \\
\hline
\end{tabular}

Fuente: PladecoPeñalolén 1993

1993 los jóvenes de 15 a 25 años cesantes alcanzan un 21,43\%, pero si se agregan los datos de jóvenes sin actividad y aquellos con quehacer en el hogar se llega a la cifra 44,5\% jóvenes de la comuna sin integración real, sin trabajo o actividades significativas, contribuyendo a engrosar las familias con pobreza dura, y componentes de cultivo para la delincuencia.
El cuadro $\mathrm{N}^{0} 4$ gráfica este proceso, de modo que comenzando en punto determinado, prácticamente se vuelve al lugar de inicio. La madre embarazada, con ciertos niveles de desnutrición, con situaciones psicológicas adversas como violencia intrafamiliar, baja autoestima, baja sociabilidad, etc. Una vez nacido el niño tienen riesgo constante de enfermedades, limitada estimulación 
psicomotora en el hogar, deprivación afectiva, etc. Estos niños pueden tener incapacidades intelectuales o sensoriales; ingresa luego al sistema educacionaly un elemento habitualmente presente es la baja condición para el aprendizaje, poca reafirmación de sus capacidades individuales, riesgo de deserción y repitencia en el colegio; posteriormente más adulto se incorpora precozmente al trabajo obteniendo bajas remuneraciones, con riesgo de entrar en la carrera de la delincuencia, la drogadicción, elalcoholismo, etc. Al establecer relaciones sexuales tempranas sin posibilidades de constituir una familia independiente, probablemente se establecen como allegados donde sus padres. Las limitaciones de espacio agravan las condiciones de desesperanza aprendida, la baja autoestima, frustración, marginalidad, no favoreciendo las posibilidades de adquirir trabajo etc. Además las precarias viviendas, impiden la intimidad, generan agresividad, violencia intrafamiliar y en definitiva ello contribuye y desestabilizar la familia, surge el alcoholismo y asi nuevamente el ciclo se repite. Obviamente este esquema es una propuesta de simulación de la realidad.

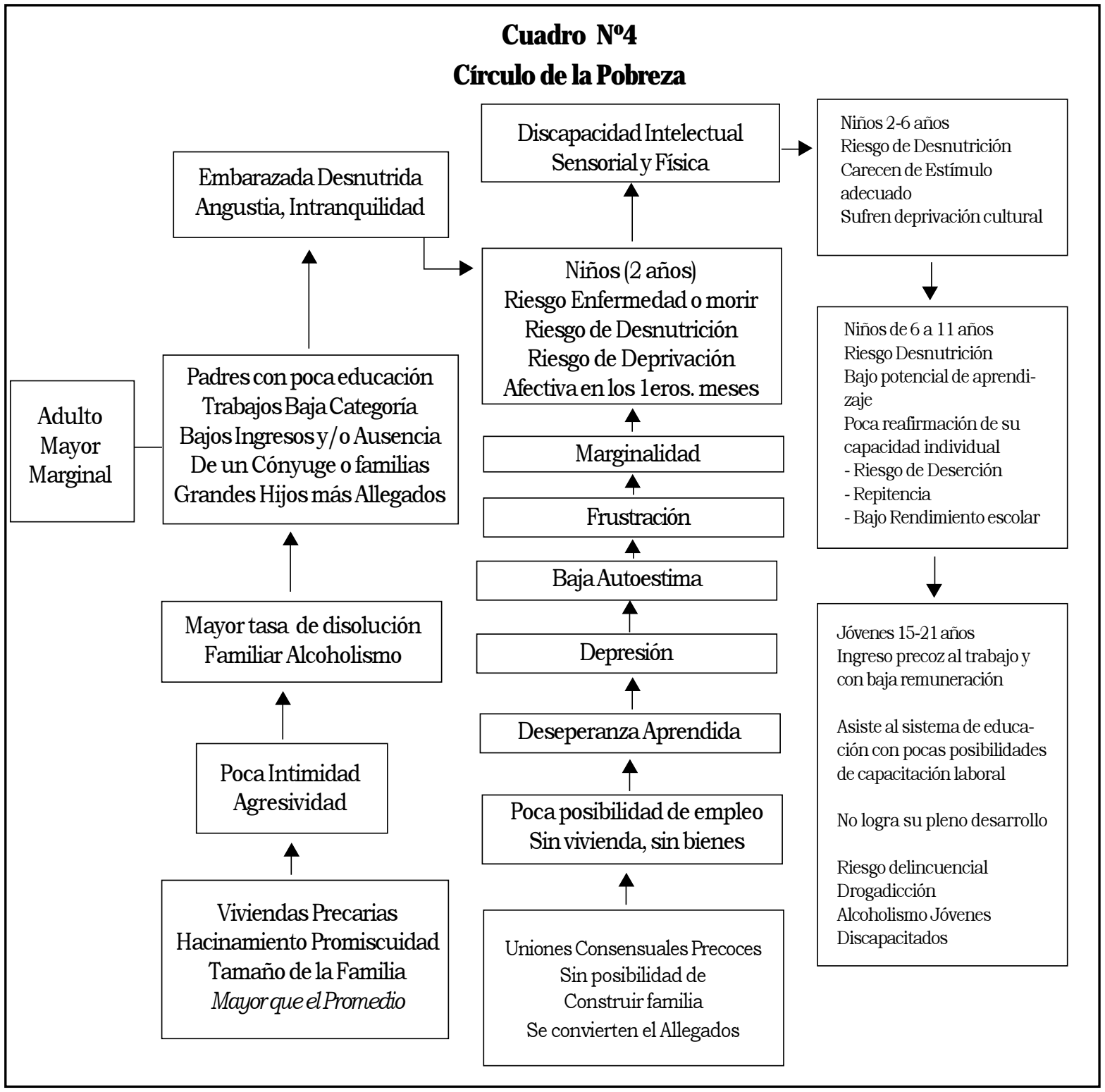


La forma habitual de explicar la pobreza dura es siempre buscando variables externas, ya sea la falta de acceso a la educación, a la salud, trabajo, etc. Por ejemplo, existen diferentes programas gubernamentales para superar la pobreza, pero son programas que en definitiva no apuntan o desconocen la realidad más intima en que viven los pobres o en que vive la familia.

Un caso al respecto es el Instituto Nacional de Desarrollo Agro-Pecuario (INDAP), que desarrolla programas de transferencia tecnológica. El campesino pobre se siente invadido por nuevas tecnologías que les ponen sobre la mesa, que no saben usar, terminan no interesándose y no participando. INDAP dirige algunos de sus programas a jóvenes (Reyes, 1998), los cuales desde una perspectiva cualitativa estos no tienen mucho éxito, fundamentalmente estos programas no logran hacer efectiva su propuesta por una falta de consideración del contextos psicosocial y cultural del campesino empobrecido.

En el campesino o la familia en pobreza dura se generan condiciones internas y psicosociales que son un "handicap" para enfrentar distintas oportunidades.

\section{IV.-Estructura Fundacional de la Familia Pobre}

Se presenta a continuación una perspectiva de la familia pobre a partir de elementos estructurantes alojados en un periodo particular de la historia de nuestro país, elementos que en términos de hipótesis hacen referencia al origen de la familia popular. El argumento inicial de esta formulación se encuentra en autores como Gabriel Salazar, Sonia Montecinos, Teresa Valdés, quienes han desarrollado un trabajo desde un enfoque básicamente cualitativo al estudiar ciertos fenómenos históricos que aluden a la familia chilena entregando asi un soporte fundacional de las variables que conforman la familia pobre.

El análisis permite observar que la calidad de fundacional radica en que son elementos que es- tán a la base y fundan un tipo de comportamiento que perdura en el transcurso de muchos años, más de un siglo, reproduciéndose de familia en familia, de generación en generación. Son elementos significativos dado que adquieren el peso en la evolución de la cultura.

Sonia Montecinos (1991) asocia la familia al proceso de conquista española, contexto en el cual la relación básica se establece entre la mujer autóctona latinoamericana, es decir la mujer indigena, con un hombre, que no es el hombre latinoamericano sino que es el español. De esta relación nace un hijo, que si es el hombre latinoamericano, el que en definitiva se transforma en un hijo sin padre, en un "guacho" por cuanto el padre español se evade, sigue su proyecto de conquista, se aleja. Queda en el imaginario social como un padre poderoso pero ausente y la estructura esencial de la familia es la mujer y este hijo sin padre. En este periodo la colonización introduce el concepto de familia monogámica occidental, la pareja constituida por madre, padre e hijos.

En este contexto ejerce una fuerte influencia y al mismo tiempo es un indicador de la forma que adopta la familia de la época, la configuración del Marianismo. La Virgen Maria es una figura venerada, valorada y recurrida bajo diferentes formas en toda América Latina. No ocurre lo mismo en Europa donde la figura importante es Jesús, que representa el reglamento, el poder, la sabiduría, en definitiva la fuerza del hombre.

Plantea Sonia Montecinos, que la imagen de la Virgen María se construye a través de un proceso sincrético donde confluyen la religión indígena y la religión católica. Su conformación cumple una relevante función social en el contexto de la época en que aparece el tipo de familia señalado, constituida por la mujer, los hijos o los guachos, con ausencia del hombre. Por un lado el Marianismo vincula dos tipos de familia y las dos culturas y por otro salva la situación negando, tapando, obnuvilando la realidad dramática de la nueva familia surgida de la conquista Española.

El marianismo, en otra dirección, influye en la conformación del ethos "Ser Madre" de la mujer 
latinoamericana. Teresa Valdés (1988) en un estudio cualitativo de 20 casos de mujeres del sector popular, logra discriminar y diferenciar tres estilos de vida en mujeres, donde uno de ellos muy significativo favorece la primacia de lo "natural". En este Modelo "la mujer es concebida como parte de la naturaleza, como parte de la madre tierra, por lo tanto debe ser fecunda y darle muchos hijos, la madre tierra posee recursos ilimitados que sus hijos, todos los que habitan en ella deben obtener. Se supone la existencia de un orden natural que resolverá los problemas de escasez que se puedan presentar".

En la constitución de una familia, desde una perspectiva natural asociado a lo religioso, el número de niños son los que Dios quiera, no es una decisión de la mujer tener uno o dos niños sino los que quiere la naturaleza, lo que el destino indique.

"Los cursos de acción que define este proyecto para la mujer se concentran en su ser madre, en una determinada versión, la mujer debe tener muchos hijos, durante todo su periodo de fertilidad, sin limitaciones de ningún tipo"... "además deberá alimentarlos, será su responsabilidad que sus hijos crezcan y sean sanos..." (pág, 168, Valdes. T, 1988)

El hombre se integra a la familia como requisito para que se cumpla el rol femenino, para que la mujer sea madre, tenga hijosy estos sobrevivan, esa es la integración del hombre en este proyecto, conforma una posición secundaria.

"El hombre se integra a este proyecto como la lluvia que permite que la semilla germine y que la alimenta para que la semilla llegue a ser una planta que posteriormente dé flores y frutos, por lo tanto debe hacer producir la tierra y proveer los alimentos necesarios para sus hijos. La mujer no está excluida de esta tarea, en última instancia es ella la responsable de la supervivencia de los hijos" (pág, 168, Valdes. T, 1988).

Sobre la pareja, "este proyecto no incluye regulaciones respecto de la pareja y si bien es necesario que la mujer tenga una pareja para que exista reproducción no define condiciones ni limitaciones es sólo un proyecto de maternidad. El matrimonio como institución y norma no es condición necesa- ria para que el proyecto se lleve acabo exitosamente, de hecho la mujer puede ser madre al margen de la normatividad social" (pág, 172, Valdes. T, 1988).

Volviendo atrás para entrar a definir el rol del hombre latinoamericano, Sonia Montecinos señala "......el mito mariano resuelve nuestro problema de origen, ser hijos de una madre india y de un padre españoly nos entrega una identidad inequivoca con una madre en común. ....Desde la óptica de las identidades de género el símbolo mariano constituye un marco cultural que asignará a las categorías de lo femenino y de lo masculino cualidades específicas". (pág, 28-29, Montecino, S, 1991)

Lo femenino es ser madre y lo masculino, es ser fundamentalmente hijo. “.... En este ámbito pensamos que el arraigo de la imaginería mariana y de sus atributos de lo Mater para lo femenino y de lo hijo para lo masculino denuncia una tensión que originada en la historia tiende a solucionarse a través de la representación que la sublima. El vacío que la expresa es de lo masculino como padre" (pág 30, Montecino, S, 1991). La incorporación del Marianismo resuelve el tema obnuvilando el rol del hombre latinoamericano como padre, dejando un vacío y donde lo masculino está sustentado por esta relación madre-hijo, expresándose fundamentalmente como hijo y luego como "huacho" y posteriormente discriminado y excluido.

Dado este vacío en el rol del hombre, Sonia Montecinos señala su compensación a través de dos vertientes, una de ellas es el caudillo, el guerrillero, es el hombre del campo que roba al patrón, el cuatrero; en el caso de la Independencia de Chile, Manuel Rodríguez cumple un rol de caudillo . El delincuente de alguna forma es un caudillo, en la literatura muchas veces figura como un héroe que logra satisfacer ciertas necesidades culturales que han sido arrebatadas. En definitiva es un hombre de la marginalidad, no perteneciente al mundo de lo formal y que se transforma en caudillo y vive como tal.

El otro camino de compensación hace referencia al machismo, este rasgo se configura a través de una concepción de superioridad del hombre por sobre la mujer, sin embargo, su expresión inmedia- 
ta es mediante la violencia. El hombre se impone haciendo uso de la fuerza en el interior de su familia, no es a través del convencimiento o de constituirse en autoridad propiamente tal. En este sentido no existe en la matriz de la familia popular el hombre como padre en equilibrio con la mujer, por lo tanto, su entrada es esencialmente por fuerza.

Otro aspecto es el tema de la conformación del guacho del siglo XIX que tiene que ver con la etapa posterior a la colonia, relacionado con el periodo del inquilinaje, con el trabajo agrícola en el mundo rural, con la oligarquia, etc. Alli la familia se encuentra en una situación especial, vive dentro de una hacienda, en un territorio donde ella es parte de las propiedades del dueño de todas las tierras y en eso la mujer nuevamente juega un rol importante. Está presente una relación entre esa mujer y el patrón; no es casual el llamado "derecho a pernada" de la época, el ascendiente sexual del patrón por sobre la mujer cuando esta contrae matrimonio. El inquilino también aquí juega un rol secundario, inferior y muchas veces frente a sus protestas es expulsado de esas tierras, sale del campo, y se transforma en gañán, o en cualquier cosa. La familia en ese periodo pasa por diferentes etapas, la familia inquilina es una de ellas.

En este periodo se encuentran antecedentes de la matriz Psicosocial de la familia pobre. El análisis histórico- cualitativo señala que la familia pobre dentro de los avatares de la época se encontraba constituida por la mujer como pilar fundamentaly los hijos, donde el hombre esencialmente jugaba un rol itinerante, y pese a que en algunos momentos surgía como elemento central también estaba ausente por largos periodos, e incluso su vuelta a casa era deseada más bien en la fantasía que en la realidad. Gabriel Salazar (1989) en su texto "Ser niño guacho en la historia de Chile del siglo XIX" expone dicha situación señalada haciendo uso de un recurso novelesco: "Esperaban ustedes otra cosa? Mateo Vega, el peón que engendró las criaturas de Rosaria no se hizo presente el día del parto, tampoco se había aparecido durante el último tiempo del embarazo....... no compadeció la angustia final de Rosaria, no se hizo cargo de ningu- no de los niños. El gobierno en consecuencia dio al padre por inexistente o por incompetente, pues en todos los documentos oficiales sólo se habló de los hijos de Rosaria Araya..... No es cosa de maravillarse, sin embargo, por el comportamiento irresponsable de Mateo Vega, porque cuando se tenía un padre como ese Mateo, es decir como un simple peón, entonces había que hacerse la idea de que papá no era sino un accidente o una cadena de accidentes en las vidas de su prole. Los hombres como Mateo no formaban familia. Se sentian compelidos más bien a andar la tierra. En camino a otros valles, de vuelta a otros fundos, en busca de otras minas. Escapando a los montes. Atravesando la cordillera. Apareciendo y desapareciendo.....Y aún podian pasar los años sin que se tuviese el menor noticiamiento de él. Hasta que alguien avisaba que estaba preso o lo habian herido en una riña de borrachos, que lo habian visto convicto o enjaulado y engrillado reparando el camino del puerto. $O$ que lo habian agarrado en una leva, que lo habian hecho servir en ejército, que se habia desertado, que en fin se había hecho cuatrero........ Asi poco a poco de pura ausencia y noticiamiento un papá del tipo de Mateo Vega, se iba transformando en la mente de sus hijos en una especie de leyenda en un padre legendario. Legendario, pero inútil. A veces admirado y deseado, pero las más de las veces temido y rechazado" (pág 5-6, Salazar, G, 1989).

Este autor describe también el contexto y la vida de la mujer de la época, quien frente un marido ausente, con muchos hijos, debiendo hacerse responsable de la sobrevivencia familiar, alcanza un nivel de saturamiento y sobrecarga que de cierta manera la obligaba a expulsar los hijos de la casa, a regalarlos para su manutención por parte de terceros. Aqui un texto de Gabriel Salazar al respecto : "Ahora ustedes dirán qué pasaba con mamá, pues como lo presintió claramente Rosaria Araya los hijos se quedan siempre aferrados a la madre, sobretodo cuando hay naufragio conyugal. Entonces digámoslo de entrada mamá se quedaba muy a disgusto con nosotros. Es que para ella no éramos más que un cepo que le impedia moverse con la presteza requerida para subsistir en un medio tan dificil 
como era el que acosaba a los chilenos pobres del siglo XIX. Donde la mayoria de los hombres -aún los más fuertes- fracasaban sin remedio viéndose obligados a escapar de sus hijos, mamá no podía escapar de nosotros. No podía. Pero, francamente, la estorbábamos. ¡Y vaya sí la estorbábamos! Si su impulso más primario - tras hecharnos al mundo y comprender que estaba sola, como Rosaria-, era repartirnos. Eso, exactamente eso: obsequiarnos a cualquier otro que sí pudiera tenernos. Ella no escapaba como papá ciertamente, pero en cambio se deshacía de nosotros tan pronto como podía. Usaban distintos procedimientos. Uno de ellos consistía en llevar al niño recién nacido, en la oscuridad de la noche, a una casona patricia, en cuyo zaguán, envuelto en toscas mantillas, se le dejaba expuesto. Ella golpeaba la puerta y escapaba" (pág, 13, Salazar, G, 1989).

\section{V.-Matrices Psicosociales de la FamiliaPobre:}

De acuerdo a los antecedentes entregados anteriormente respecto de las matrices fundacionales de la familia es posible esbozar en términos de hipótesis una propuesta sobre los elementos estructurales básicos de la familia pobre en nuestro medio.

Hay un conjunto de características que describen la familia pobre según lo señalado, estos elementos pudieran aparecer como teñidos por un sesgo negativo, no obstante no es la idea que se quiere presentar.

La familia pobre se configura sobre dichos aspectos de origen, pero la hipótesis avanza en el sentido que en el desarrollo presente de la misma, estos elementos se acentúan y se sobredimensionan. Este resultado sería fruto de dos factores relevantes, por un lado, el enfrentamiento de la familia pobre a la modernidad, dinámica que implica tal nivel de exigencia que esta no puede absorber, empujándola a un mayor nivel de deterioro. Este mismo proceso hace surgir el segundo factor, la pobreza dura, condicionando un tipo de familia gravemente desarticulada.

El tema de la modernización está relacionado con la urbanización, con el traslado masivo de la población rural al mundo urbano desde 1930, de modo que aproximadamente el $80 \%$ de la población de nuestro país y en Latinoamérica ya vive en la ciudad (Rozas, 1998). El tipo de familia diseñada arriba, sujeto a las matrices fundacionales señaladas llega a la ciudad y se encuentra con que el mundo urbano no está pensado para ella sino para un tipo de familia europea, nuclear, con padre, madre e hijos. Quedan marginados abuelos y allegados. Concepto de familia occidental que promueve un mayor equilibrio hombre-mujer, centrada en la educación de los niños, condicionando una sexualidad basada en el control de la natalidad, valorando un número de hijos reducido. La familia descrita arriba se golpea en esta modernidad, su fracaso implica deterioro, procesos de expulsión, salida temprana del hogar, prostitución.

A continuación se presenta un diagrama que expresa el conjunto de caracteristicas de la familia asociada a la pobreza dura inmersa en una relación de circularidad, en que cada miembro de la familia desarrolla un rol entropico, desestructurante. 


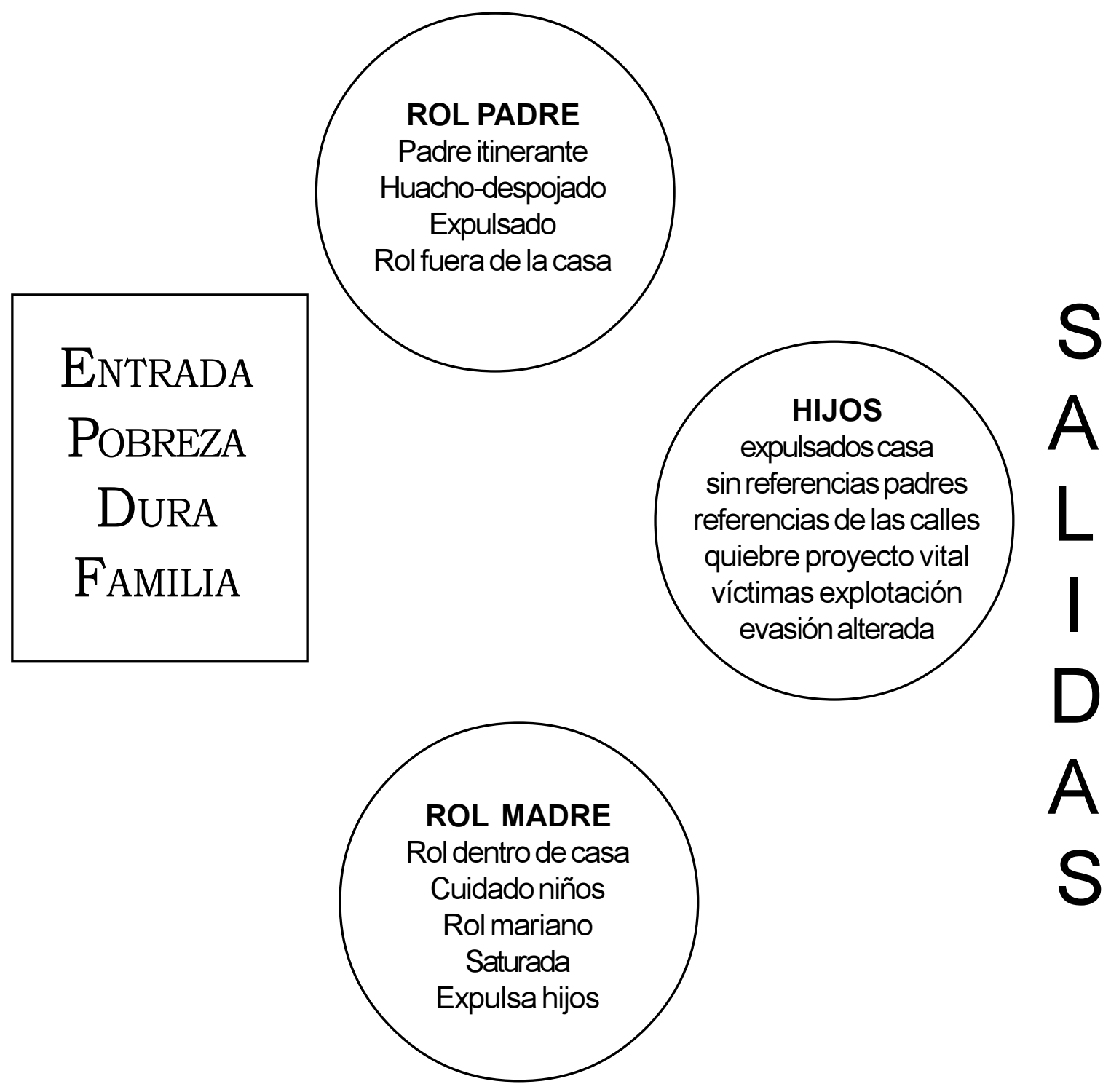

VARIABLE EXTER:

Migración

Modernismo

Segregación urbana Inequidad 
Finalmente a continuación se expone los diferentes roles de la familia, entendiendo por esta a una familia en pobreza dura.

\section{LaMujer}

Las familias pobres tienen un porcentaje mayor de Jefatura femenina, que otras familias, la inestabilidad del cónyuge en la vivienda empuja a la mujer a asumir la responsabilidad total del hogar. Más aún la mujer, en referencia a su cónyuge, vive una relación contradictoria en la cual muchas veces lo rechaza y se esfuerza por su salida de la casa en tanto existe violencia, alcoholismo y cesantia.

Ser madre es la caracteristica principal presente en la subjetividad de la mujer, sin embargo, ello es posible en tanto el cónyuge hace un aporte financiero al hogar, alli la mujer asume la responsabilidad de la sobrevivencia de los hijos.

Sin embargo el cúmulo de preocupaciones, de obligaciones, de urgencias, de múltiples necesidades genera en la mujer una sobresaturación, cansancio, agotamiento y numerosas frustraciones. Esta situación produce una presión hacia sus hijos por la búsqueda del sustento, en algún momento la madre en relación a sus hijos, prioriza más el trabajo, el logro de algún tipo de ingreso que el estudio. Lo que en la práctico, sin ser una actitud consciente implica una presión hacia el alejamiento de los hijos del hogar. De esta manera se va gatillando la independencia temprana de los hijos.

\section{ElPadre}

En el caso del padre, pero más bien padre itinerante, tiene que ver con los elementos fundacionales de la cultura chilena. El constructo simbólico de "guacho" forma parte de los arquetipos del hombre popular. Si bien puede no corresponder a su realidad actual, forma parte de su historia familiar o es vivenciada a través de la generación de sus pares
Vivir dentro y fuera del hogar, pareciera una actitud ambivalente ante la vida, sin embargo, el hombre popular se encuentra proyectado hacialo exterior, fuera de la casa o cercano a la vida de la calle, al campo. Si la situación del hogar se complica resuelve sus dificultades alejándose, tomando distancia. Se encuentra orientado a tratar sus problemas en espacios del exterior con otros, en el bar, en la plaza, en la cancha de fútbol.

Por otro lado su condición de pobre, producto de su condición social, sin inserción social, falente de capacitación, lo deja sometido al riesgo constante de la explotación, del despojo, de bajas remuneraciones. Cuando la crisis familiar se dispara, generalmente por necesidades económicas, el padre asume de forma ambivalente, dinámica en la cual por periodos se encuentra en el hogar y otros fuera del mismo. Desconociendo la crisis retirándose, actuando de manera ambigua, se diria que actúa como un hijo que deja los problemas a su madre.

Es claro que este patrón de comportamiento no nace del padre en cuestión sino que se encuentra a la base en tanto sustrato historico-cultural y soporte inequivoco del despliegue de la familiar pobre en los momentos de crisis.

\section{Los Hijos}

Luego, el patrón de comportamiento de los hijos expulsados a edades tempranas. El marco de referencia es la calle. Si sale de la casa tempranamente el niño se encuentra con jóvenes, con grupos o pandillas, aprende de las situaciones dramáticas presentes en la calle, delincuencia, drogadicción, alcoholismo, etc. Aprende de alguna forma a vivir en esta selva. Muchas veces víctima de la explotación, de vivir al dia, muchos niños desarrollan una concepción de mundo castrada, acentuando más el presente que el futuro.

Al respecto las referencias parentales son limitadas, ambos padres no alcanzan a configurar modelos de desarrollo, en tanto cuanto, sus respectivos proyectos vitales y sus roles al interior de la familia se encuentran trizados. 


\section{ReflexionesFinales:}

La Pobreza Dura constituye una realidad muy impactante que afecta a grandes sectores de población. Producto de variables estructurales, particularmente de indole económicos, la pobreza dura se expresa más allá de esta dimensión configurándose como un fenómeno esencialmente cualitativo de carácter psicosocial.

Adquieren aquí su máxima importancia variables como la desesperanza aprendida, la baja autoestima, deterioro en el proyecto de desarrollo vital, la transmisión intergeneracional de la pobreza.

El efecto de esta realidad sobre la familia es devastador, por cuanto la subsume en un círculo perverso que profundiza sus limitaciones y obstaculiza sus recursos.

La familia conforma un espacio social de desarrollo para sus integrantes. Sin embargo, en tanto fenómeno social no está libre de ser parte de un proceso de evolución histórica. Dinámica que pone sobre el tapete características particulares de la familia pobre, de la familia campesina. Elementos que de una y otra forma, conforman un sustrato básico de desarrollo de la misma y que tienen fuerte incidencia en la evolución de la familia hoy en día.

A este sustrato es lo que se ha aludido como matrices fundacionales de la familia pobre. Ahora, nuestro análisis nos orienta a combinar dichas matrices con el tema de la pobreza. Dicha dinámica de conjunción, de encuentroy amalgama devela, la expresión de la pobreza en la familia como pobreza dura.

Matrices como el rol de la mujer fundamentalmente como madre, o del hombre básicamente como hijo, externo, proveedor distante, etc. son factores que impactan en el desarrollo de los hijos quienes cuestionan al poco andar la autoridad de sus padres autonomizandose de su hogar, en equivocas condiciones que los dejan expuestos y vulnerables a estímulos disruptivos del entorno.
Se produce un círculo vicioso que reproduce condiciones psicosociales autolimitantes frente al desarrollo. Queda evidente así que la pobreza no es sólo una cuestión económica, especialmente en elámbito de la pobreza dura.

Se quiere insistir que la propuesta realizada constituye más bien una hipótesis, en la expectiva de desarrollar una discusión en elámbito de la psicología social sobre el fenómeno de la familia en el contexto de la pobreza dura.

Por otro lado aquí ha quedado en evidencia fundamentalmente carencias y dimensiones críticas de la familia pobre. No puede inferirse, sin embargo de este planteamiento, que dichos aspectos sean los únicos presentes en la misma, constituyen sólo una de sus facetas. No cabe duda de las enormes cualidades y riquezas presentes en nuestra familia popular como ya se ha señalado (Rozas, 1992) y que se requiere continuar profundizando.

\section{Bibliografia}

Comisión Económica para América Latina y el Caribe (1994) "Panorama Social de América Latina" CEPAL, Santiago de Chile Instituto Nacional de laJ uventud (INJ ), (1994) "Primer Informe Nacional de Juventud" Santiago de Chile

Ministerio de Planificación y Cooperación (1994) Dpto. de Planificación y Estudios Sociales. Encuesta Cases 1990 y 1992, MIDEPLAN, Santiago de Chile

Ministerio de Planificación y Cooperación (1997) "Pobrezay Distribución del Ingreso en Chile, 1996. Resultados de la Encuesta de Caracterización Económica Nacional" MIDEPLAN, Santiago de Chile

Montecino, S. (1991) Madres y Huachos. Alegorias al Mestizaje Chileno Ed. Cuarto propio-CEDEM, Santiago de Chile.

Plan de Desarrollo Comunal, PLADECO (1993), Comuna de Peñalolén, Santiago de Chile

Reyes, Paula. (1998) "Politicas Sociales hacia Jóvenes. Una descripción metodológica de la medición del impacto comunitario" Memoria, Dpto. de Psicologia, Universidad de Chile.

Rozas, G. (1998) "Psicologia Comunitaria, Ciudad y Calidad de Vida", Revista de psicologia, Vol VII, Dpto de Psicologia, Universidad de Chile.

Salazar, Gabriel (1989) "Ser niño huacho en la Historia de Chile (siglo XIX)" Ponencia presentada al Seminario "sociedad Agrícolay Minera Chilenas en la Literatura y en la historia", del Dpto. de Historia de la Universidad de Santiago, Santiago de Chile.

Valdes, T. (1988) "Venid, benditas de mi Padre. Las Pobladoras, sus rutinas y sus sueños” FLACSO, Santiago de Chile. 